\title{
Justification and calculation of design features of drum-type hydroponics
}

\author{
A.A. Sadov", A.D. Ustyugov, I.L. Chukin, K.M. Potetnya, and M.V. Kholmanskikh \\ Ural State Agrarian University, Department Technological and transport vehicles of the faculty of
} engineering technology, Yekaterinburg, Russia

\begin{abstract}
The purpose of the article is to evaluate the design features of rotary hydroponics and then obtain a method for calculating the main technical characteristics. Drum (rotary) hydroponics is a promising method of growing on artificial media. The planting surface in this method is located on the inner cylindrical surface of the rotor rotating around the lamp. The design of the rotary hydroponics has a number of advantages such as: smaller installation area due to planting in the cylinder area; energy savings due to rational use of the light source; liquid saving for the formation of a nutrient solution due to the alternate immersion of the roots in a more compact bath. In this paper, a number of theoretical aspects for the calculation of drum (rotary) hydroponic machines is considered to ensure the highest productivity. As a result, a method for calculating the following characteristics was derived: determination of the required radius of the rotor depending on plant height; number of planting spots with a known radius and width of the rotor; minimum amount of necessary nutrient solution; electricity consumed when using LED lamps.
\end{abstract}

\section{Introduction}

Hydroponics is a method of plant growing on artificial media instead of soil. Nutrition is carried out by means of a nutrient solution supplied directly to the root system. Hydroponics provides ample opportunities for regulation of growing conditions. Optimal conditions ensure that high yields of high quality are obtained in the shortest possible time.

At the moment, there are the following types of hydroponic systems:

- $\quad$ Periodic flooding system;

- Deep-water crop system;

- Nutrient layer system;

- $\quad$ Drip irrigation system;

- Aeroponics system.

- Wick system;

\section{Literature Review:}

In most enterprises, the plant cultivation by hydroponics method is carried out in a rack method. (Fig. 1). This method is the least demanding of the equipment, it can be used both at

\footnotetext{
* Corresponding author: artemsadov@ya.ru
} 
home and on an industrial scale, but in this case it has a number of disadvantages. The main one is the use of large areas. This problem entails a number of other problems, such as the laying of long water supply lines, heating, ventilation and lighting of large rooms.

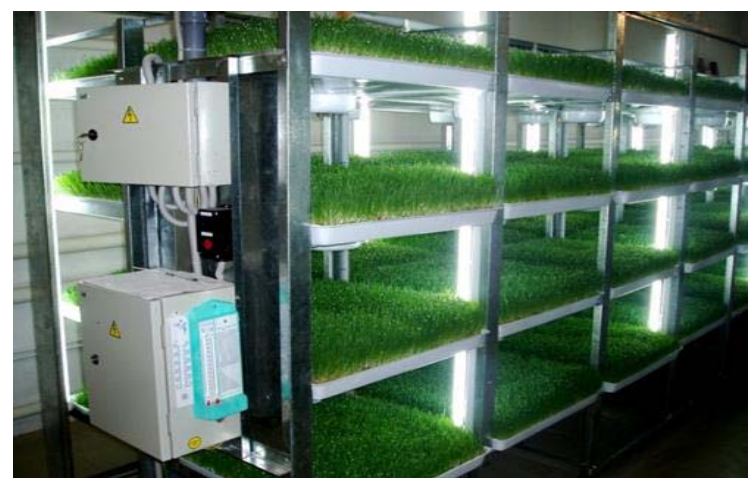

Fig. 1. Rack hydroponics.

Rotor hydroponics is a special approach to the periodic flooding system. The design is based on a rotor rotating around the light source, the planting area is located on its inner cylindrical surface (Fig. 2).

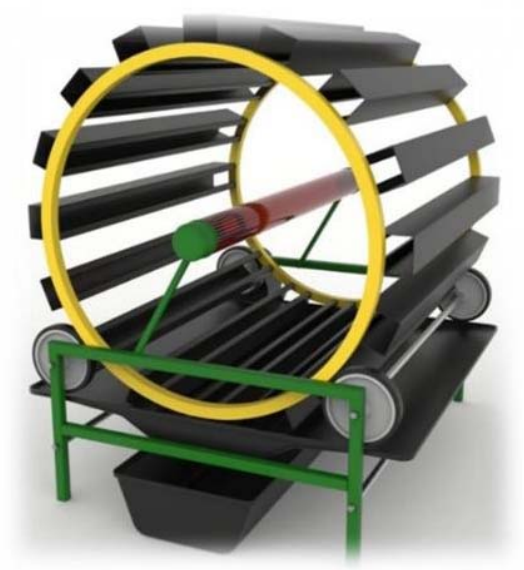

Fig. 2. Rotor hydroponics.

\section{Materials and Methods}

The study used theoretical methods based on research the main scientific and technical literature and theoretical studies, the application of the provisions of trigonometry and mechanics with verification in laboratory conditions on a prototype of rotary hydroponics.

\section{Results and Discussion}

Space saving is the main advantage of rotor hydroponics. It is clearly demonstrated by the example of the ratio of the rotor planting surface area $S_{\text {rot }}$ to the area on which the rotor hydroponic machine is placed $S_{p l}$ : 


$$
S_{p l}=a b
$$

where $a$ - width, $b$ - length of the placement zone.

The rotor potentially placed on this area can reach up to the width of the placement area in the outer diameter $a$. The planting surface is located on the inner diameter of the rotor. The ratio of the rotor outer diameter to the inner diameter is determined by the design features and the crops grown. For example, take the inner diameter $d=0,6 a$. At the same length $b$, the rotor growing area $S_{\text {rot }}$ will be:

$$
\begin{aligned}
& S_{r o t}=(\pi d) b=1,9 a b \\
& \frac{S_{r o t}}{S_{p l}}=\frac{1,9 a b}{a b}=1,9
\end{aligned}
$$

The area ratio in this case will be almost the twofold advantage compared to rack hydroponics, which is not the limit. The combination of certain design features may exceed this indicator.

As for the height, the entire rotor surface, its lower and upper points, are illuminated by a single lamp, which also provides additional compactness, while the racks with separate lamps on each tier are stacked on top of each other.

However, with the above prospects, rotor hydroponics has a number of features and sets certain tasks for the development of the design and algorithms for growing, such as: restrictions on the rotor size; characteristics of the lighting source for the most optimal light distribution; optimal frequency of rotor rotation; influence of these parameters on the plant growth.

The machine diagram is shown in Fig. 3. The internal space of the machine is closed, but in the diagram, it is represented as open for the purposes of clarity. Pots with quick-release mounting are installed in the rotor (A), rotating by an electric drive around the lamp (B). At the bottom of the rotor there is a bath with a nutrient solution $(C)$, into which the substrate in the pots and the plant roots sprouting in it are immersed during rotation. The bath is connected to the solution node (D), from which the nutrient solution enters it. Solution node is a container that receives clean water from a tank (E) or from a water pipe through a filter, as well as nutrients from the corresponding containers $(\mathrm{F})$. After receiving the required amount of water and nutrients, the sensors installed in the solution node measure the characteristics of the resulting solution and adjust it if necessary. Also, in the node, the solution is brought to the optimal temperature, after which it begins to circulate between the solution node and the bath. The waste solution and excess liquid are drained into the waste tank $(\mathrm{G})$ or directly into the sewer, depending on the configuration. The temperature and humidity of the interior space are also measured and maintained within the necessary limits due to ventilation, heating, and cooling systems. 

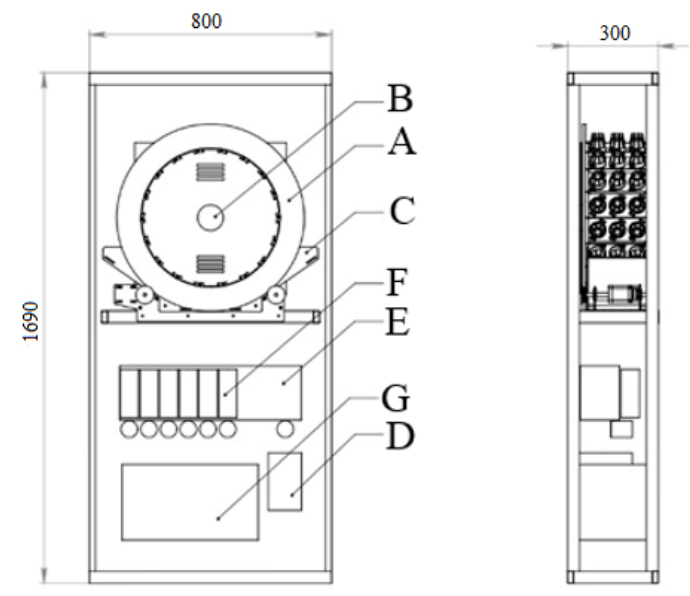

Fig. 3. Machine diagram: A - rotor, B - lamp, C - bath, D - solution node, E - tank with clean water, F - containers with fertilizers, $\mathrm{G}$ - tank with waste.

The machine control is carried out through the developed graphical interface, accessible to the basic user. The indicated dimensions are preliminary, but they reliably reflect the overall characteristics of the final product.

The hydroponic method provides rapid growth and rapid crop loss in case of violation of the established growing process. To protect against abnormal situations and software failures, the machine is equipped with an uninterruptible power supply. In case of complete blackout, the UPS will maintain the rotor rotation and the wetting of the plant roots even if all other functions are interrupted, which will save the crop until the power supply is restored. The level of the nutrient solution in the bath is maintained at a fixed level due to the design features and is also independent of the electricity and water supply.

Figure 3 shows a working version of a small rotor hydroponic plant and a $3 \mathrm{~d}$ model of the concept of the developed machine with the solution node and full automation.

The number of planting spots in the rotor can be determined empirically. Nevertheless, when developing new configurations of the rotor hydroponic machine, as well as evaluating the planting areas in case of industrial applications of rotor hydroponics, a preliminary calculation may be necessary.

As the initial data, we will need the following values:

$R_{r}$ - radius of the rotor planting surface;

$d$ - diameter of the planting hole of the pot used;

The radius of the rotor planting surface $R_{r}$ can also be calculated with the lamp radius $r_{\text {lamp }}$ and the height laid down for the grown plantsh $h_{\text {plant }}$ :

$$
R_{r}=r_{\text {lamp }}+h_{\text {plant }}
$$

Each spot is occupied by a circular segment bounded by a chord. Thus, the number of spots per radius of the rotor will be:

$$
n=\frac{c}{l}
$$

where $n$ - the number of spots (pots) per cross-section $C$ - the circle length, $l$ - the segment arc.

Developing this formula through the disclosure of the circle length we receive:

$$
n=\frac{2 \pi R_{r}}{l}
$$


The calculation scheme is shown in Fig. 4.
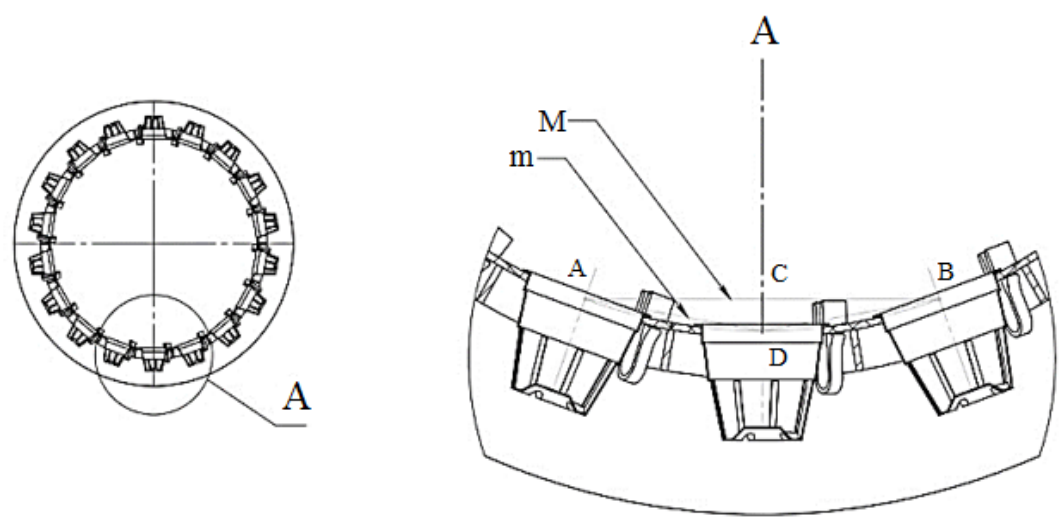

Fig. 4. Rotor cross-section diagram.

To find the segment arc, the Huygens formula is used:

$$
\begin{gathered}
L_{A D B}=2 l=2 m+\frac{1}{3}(2 m-M) \\
l=\frac{1}{2}\left(2 m+\frac{1}{3}(2 m-M)\right)
\end{gathered}
$$

where $m$ is the chord $\mathrm{AD}$ and $\mathrm{DB}, M$ is the chord $\mathrm{AB}$.

It is worth considering that the relative error of the formula is $0.5 \%$ when the arc $\mathrm{AB}$ angle is $60^{\circ}$. When the angle decreases, the percentage drops and for an angle of $45^{\circ}$ it is already $0.02 \%$.

The chord $m$ is the distance between the intersection points of the axes of the holes with the cross-section circumference. Or the length of the area occupied by the pot diameter $d$ and offset $a$.

$$
m=d+a
$$

The value $a$ is the interval that extends to the interval between the potholes in the rotor. The rotor surface holes can not physically be placed close together, therefore, the size $a \neq 0$ depends on the design features of the rotor.

The chord $M$ can be obtained empirically in the presence of the initial value of the rotor radius $R_{r}$ after value $m$ calculation.

Thus, the number of spots per rotor cross-section $n$ will be:

$$
n=\frac{4 \pi R_{r}}{2 m+\frac{1}{3}(2 m-M)}
$$

The obtained result should be rounded down.

The rotor width $H_{r}$ can be determined by the formula:

$$
H_{r}=d N+b_{1}(N-1)+2 b_{2}
$$

where $d$ - the diameter of the planting hole of the pot used, $N$ - the number of rows along the rotor rotation axis, $b_{1}$ - the offset between the holes along the rotor rotation axis, $b_{2}$ - the offset from the extreme planting holes to the ends of the rotor (may differ from $b_{1}$ ). The geometric meaning of the stated values is clearly shown in Fig. 5. 

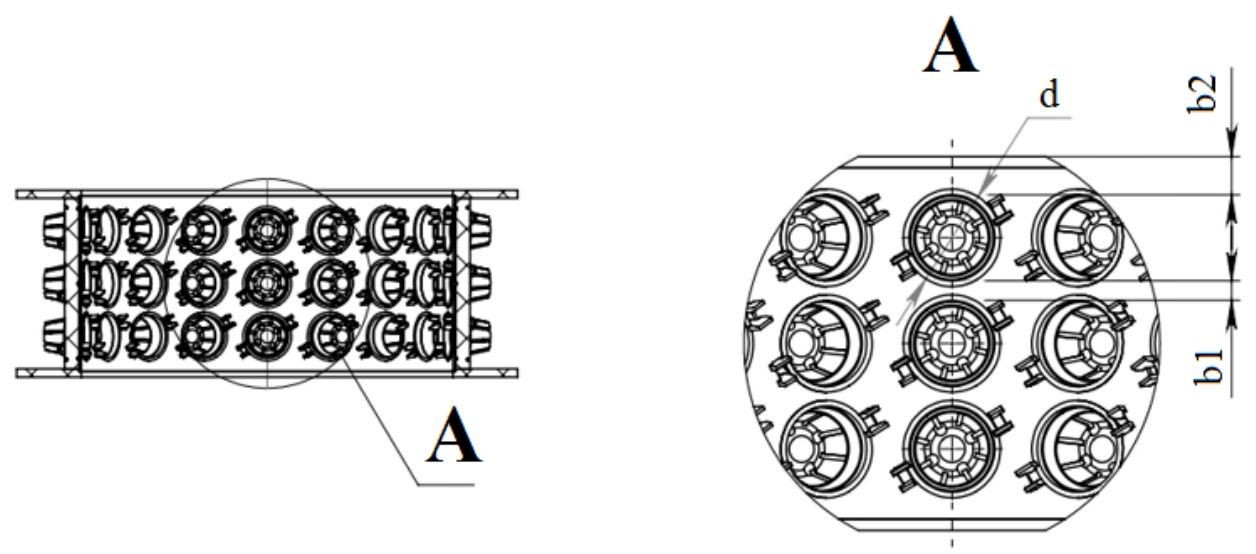

Fig. 5. Longitudinal diagram of the rotor.

By converting the formula for calculating the rotor width $H_{r}$, we get the formula for calculating the number of rows along the rotor rotation axis $N$ :

$$
N=\frac{H_{r}+b_{1}-2 b_{2}}{d+b_{1}}
$$

The obtained result should be rounded down.

In total, the total number of spots will be $N_{\text {total }}$ :

$$
N_{\text {total }}=n * N
$$

where $n$ is the number of spots per cross-section, and $N$ is the number of spots along the rotor rotation axis.

It is worth noting that the formulas for calculating the number of spots is relevant for the linear arrangement of rows along the rotor rotation axis. When shifting the rows to arrange the spots in a staggered order, it is necessary to divide them into groups located relative to each other linearly along the rotor rotation axis. The calculation is made for these groups separately, after which the result is summed up.

As for the number of spots, the dimensions of the bath and the minimum volume of the nutrient solution can be determined empirically. Nevertheless, when developing new configurations of a custom rotor hydroponic machine, as well as estimating planting areas in case of industrial applications of rotor hydroponics, a preliminary calculation may be necessary, the formulas for which are derived in this section.

As the initial data, we will need the following values:

$R_{r}$ - radius of the rotor planting surface;

$d$ - diameter of the planting hole of the pot used;

$h$ - height of the pot used;

The bath volume will depend on its design features and available technical means of manufacture. Nevertheless, to calculate the minimum amount of nutrient solution consumed, it is necessary to take a shape with a cross-section in the form of a circle segment, the center of which coincides with the center of the rotor circle. Thus, the bath $R_{v}$ radius will be:

$$
R_{v}=R_{r}+h+c
$$

where $c$ is the depth of the bath below the pot bottom required for the root system. This value depends on the characteristics of the crops grown in this machine. 
To find the volume, we need the segment area. As we can see from the diagram in Figure 6, the segment $S_{\text {seg }}$ area will be:

$$
S_{\text {seg }}=S_{\text {sec }}-S_{\triangle O B C}
$$

By converting this formula, we get the area of the segment $S_{\text {seg }}$ through the anglea:

$$
S_{\text {seg }}=\frac{1}{2} R_{v}^{2}(a-\sin a)
$$

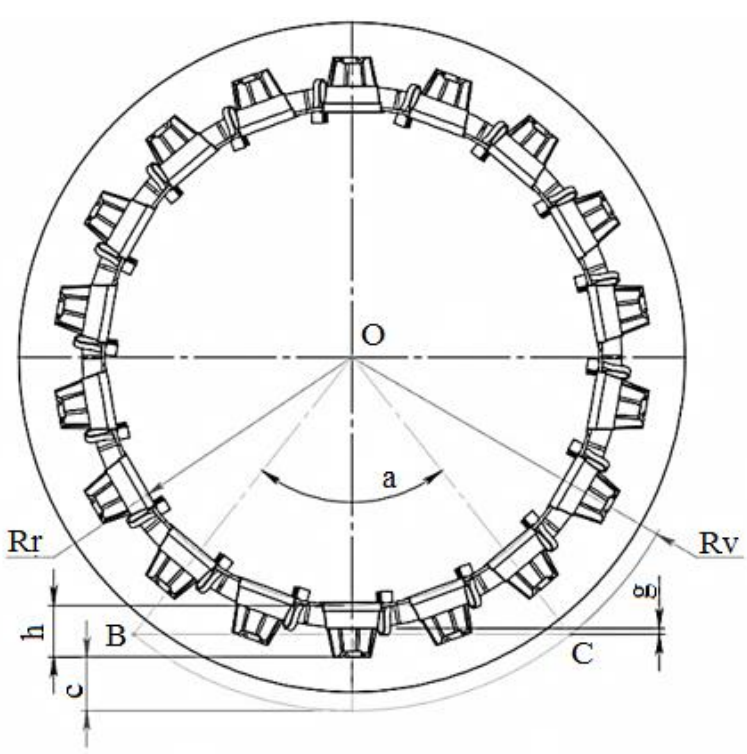

Fig. 6. Design scheme of the bath.

The angle $a$ is obtained by converting the segment height formula $h_{\text {seg }}$ :

$$
\begin{aligned}
& h_{s e g}=R_{v}\left(1-\cos \frac{a}{2}\right) \\
& a=2 \arccos \left(1-\frac{h_{\text {seg }}}{R_{v}}\right)
\end{aligned}
$$

The height of the segment $h_{\text {seg }}$ in this case:

$$
h_{\text {seg }}=h+c-g
$$

where $g$ is the structural gap laid between the rotor and the bath.

Thus, the area of the segment $S_{\text {seg }}$ will be:

$$
S_{\text {seg }}=\frac{1}{2} R_{v}{ }^{2}\left(2 \arccos \left(1-\frac{h_{\text {seg }}}{R_{v}}\right)-\sin \left(2 \arccos \left(1-\frac{h_{\text {seg }}}{R_{v}}\right)\right)\right)
$$

The bath width formula $H_{v}$ is like the rotor width formula $H_{r}$ :

$$
H_{v}=d N+b_{1}(N-1)+2 b_{3}
$$


where $d$ - the diameter of the planting hole of the pot used, $N$ - the number of rows along the rotor rotation axis, $b_{1}$ - the offset between the holes along the rotor rotation axis, $b_{3}$ - the offset from the extreme planting holes to the ends of the bath (may differ from $b_{1}$ ).

Thus, we have all the values for calculating the minimum volume of the bath $V_{v}$ :

$$
V_{v}=S_{s e g} H_{v}
$$

The minimum volume of the nutrient solution $V_{p r}$ is not limited to the volume in the bath, it also includes the volume circulating in the solution node and pipelines $V_{r y}$.

$$
V_{p r}=V_{v}+V_{r y}
$$

\section{Lighting and lamps}

One of the key elements in the hydroponic growing method is artificial lighting. For the most effective crop growth, it must meet a number of parameters, such as the placement, temperature of the lamps, the light spectrum, the correspondence of the lighting mode to the growth stage and cycle, and the coverage area.

Some of these parameters are controlled programmatically according to proven algorithms, while others depend on the design features of the machine. The latter include the following:

- $\quad$ Placement above plants;

- Lamp temperature and cooling;

- Coverage area;

One of the main advantages of rotor hydroponics is the rational use of the light source. The planting surface is located on the cylindrical surface of the rotor rotating around the lamp. Thus, the lighting is evenly distributed over the entire $360^{\circ}$, and the light source is set at the same distance from all the plants, as shown in Figure 7. This design feature leads to significant energy savings.

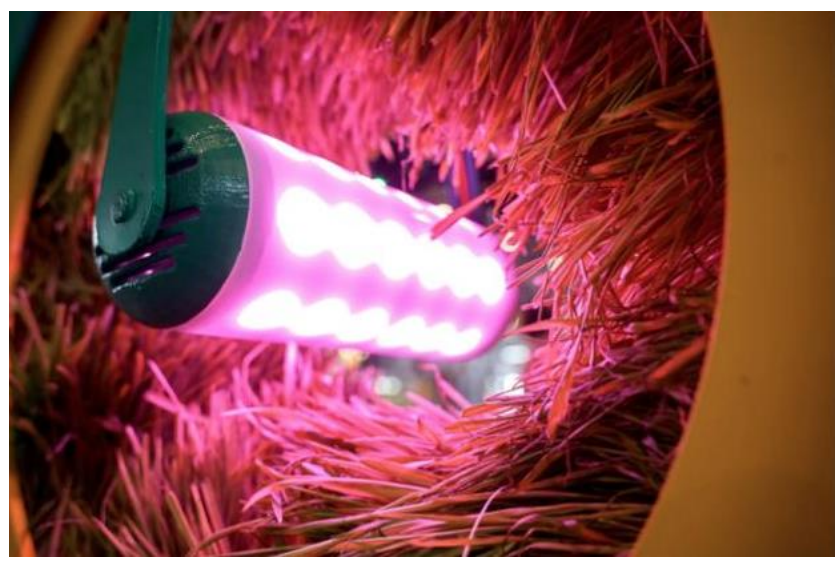

Fig. 7. Lamp in rotor hydroponics.

Nevertheless, with the above advantages, different stages of one plant growth may have different optimal distance to the light source. To achieve the maximum growth rate, this nuance in rotor hydroponics can be solved in one of the following ways: the use of replaceable lighting modules, the development of a lighting module with a variable distance to the planting surface, as well as the preliminary cultivation of plants in a separate chamber [6]. 
At the same time, the proximity of the light source to the plants imposes special requirements on the type of lamps and their heating temperature. The least heating is provided by LED lamps. Their design features make it convenient to use forced cooling, reducing heating to a minimum.

In this machine, the lamp design consists of LED strips located along the cylindrical surface rotation axis (Fig. 8).

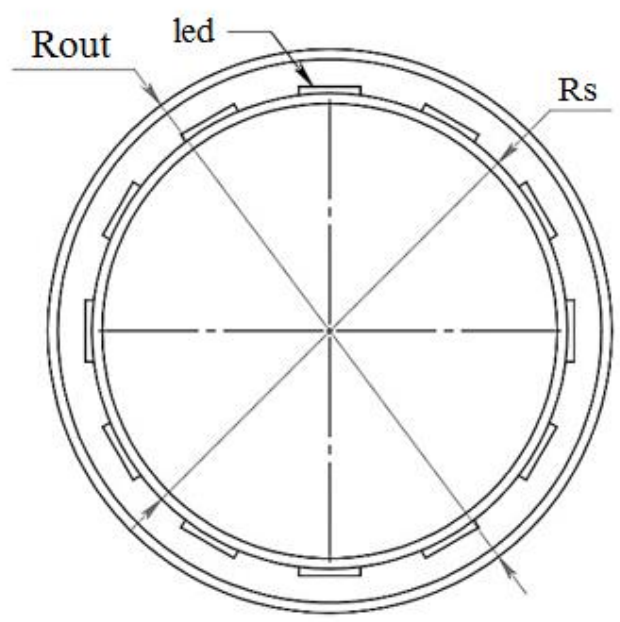

Fig. 8. Lamp diagram (cross-section).

The distance between the LEDs on the lamp cross-section (the number of LED strips) is determined as follows:

$$
L_{s}=\frac{C_{s}}{n_{s}}
$$

where $L_{s}$ is the arc length per strip, $n_{s}$ is the number of LED strips. $L_{s}$ can not be less than the width of the LED strip.

The number of LED strips $n_{s}$ at this stage is determined empirically. The calculation of the number of LEDs per lamp and rotor cross-section is the subject of a separate study, the results of which can be derived from the corresponding selection tables.

The circle length of the base surface of the lamp $C_{s}$ on which the LEDs are placed:

$$
C_{s}=2 \pi R_{s}
$$

where $R_{S}$ is the radius of the base surface of the lamp.

The number of LEDs $n_{L E D}$ depends on the number of LED strips $n_{s}$ and their length $l_{L E D}$, the formula for calculating which:

$$
l_{L E D}=d N+b_{1}(N-1)+2 b_{4}
$$

where $d$ - the diameter of the planting hole of the pot used, $N$ - the number of planting rows along the rotor rotation axis, $b_{1}$ - the offset between the holes along the rotor rotation axis, $b_{4}$ - the offset from the extreme planting holes to the ends of the lamp (may differ from $b_{1}$ ). The geometric meaning of this formula is clearly revealed in the chapter "The number of planting spots and rotor characteristics".

Number of LEDs per strip $n_{L E D S}$ : 


$$
n_{L E D s}=\frac{l_{L E D}}{h_{L E D}}
$$

where $h_{L E D}$ is the LED pitch on the strip.

Calculation of the total power of LEDs:

$$
P=\frac{U I_{1} n_{L E D}}{K}
$$

where $U$ - supply voltage, $\mathrm{V}$

$I_{1 L E D}$ - current of one LED, A

$n_{L E D}-$ total number of LEDs

$K$ - coefficient that considers the number of LEDs connected in series

The calculation of the electricity consumed $Q$ in $\mathrm{kW} / \mathrm{h}$ is made according to the following formula:

$$
Q=\frac{P t}{1000}\left(\frac{k W}{h}\right)
$$

where $P$ is the total power of the LEDs (W), $t$ is their turn-on time (h).

Lighting in hydroponics is one of the main electricity consumers. The above formulas allow to estimate the daily costs of a rotor hydroponic machine.

\section{Conclusion}

In this paper, a number of design features of rotor hydroponic machines are considered. A number of formulas are derived that make it possible to make a preliminary assessment of the design parameters of the rotor hydroponic machine or an industrial complex based on their application. There are the dimensions of the rotor, the number of spots, the minimum volume of the solution and the cost of electricity among these characteristics.

\section{References}

1. V.I. Vasilenko, A.V. Belousov, V.I. Kulish, A.A. Belousov, World of innovations. 1-4, 32-36 (2015) URL: https://e.lanbook.com/journal/issue/302388 (date of request: 17.11.2020)

2. Yu.A. Berkovich, N.V. Zyablova, A.N. Erokhin, S.O. Smolyanina, N.M. Krivobok, Aerospace and environmental medicine, 4, 54-59 (2007)

3. D. Touliatos, I.C. Dodd, M. Mcainsh, Food and Energy Security, 5(3), 184-191 (2016)

4. D. Despommier, Journal fur Verbraucherschutz und Lebensmittelsicherheit, 6(2), 233$236(2011)$

5. C.a Gómez, R.C.b Morrow, HortTechnology, 23(1), 93-98 (2013)

6. Heller, H.a , Bar-Tal, A.a, Assouline, S.a , Narkis, K.a, Suryano, S.a, De La Forge, A.b , Barak, M.c , Alon, H.d , Bruner, M.d , Cohen, S.d , Tsohar, D.d, Irrigation Science, 33(1), 53-65 (2015)

7. G. Linsley-Noakes, L. Wilken, S. De Villiers, Acta Horticulturae, 708, 365-370 (2006)

8. Agro Grow Technology: a hydroponic revolution with the Rotary Garden https://www.healtheuropa.eu/agro-grow-technology-a-hydroponic-revolution-with-therotary-garden/95655/ (date of request: 17.11.2020). 
9. Design and Development of a Solar Hydroponic Rotary Cropping Apparatus with an Intelligent Irrigation System https:/www.researchgate.net/publication/328838489 Design_and_Development_of_a_Solar_Hydroponic_Rotary_Cropping_Apparatus_wi th_an_Intelligent_Irrigation_System (date of request: 17.11.2020).

10. A.N. Musin, A.A. Sadov, A.I. Noskov, Scientific and Technical bulletin of technical systems in the Agroindustrial Complex, 2(7), 68-74 (2020)

11. A.A. Sadov, P.N. Shorokhov, M.L. Yusupov, A.N. Zelenin, Requirements for rotor hydroponics, Institutional transformations of the Russian agro-industrial complex in the context of global challenges. Collection of abstracts based on the materials of the III International Conference. Resp. for the issue A.G. Koshchaev, 105 (2019).

12. A.A. Sadov, K.M. Potetnya, A.I. Noskov, Scientific and Technical bulletin of technical systems in the Agroindustrial Complex, 3(3), 39-45 (2019)

13. A.A. Sadov, A.I. Noskov, D.O. Volkov, Scientific and technical bulletin of technical systems in the Agroindustrial Complex, 4(4), 50-56 (2019) 